\title{
The FeelMouse: Making Computer Screens Feelable
}

\author{
W. Kerstner, G. Pigel, M. Tscheligi \\ Vienna User Interface Group University of Vienna \\ Lenaug. 2/8, 1080 Vienna \\ \{gp, mt\}@ani.univie.ac.at
}

\begin{abstract}
The FeelMouse supports the user by providing tactile and force feedback to the user. Depending on the object the user points to the force necessary to press the mouse button differs. With more or less resistance the user perceives harder or softer objects or objects which jut out more or less from the virtual computer surface. If the force is switched continuously a vibrating object is generated. In addition to "normal" usage the FeelMouse can be used to enable the work with GUI's also for visually impaired people.
\end{abstract}

\section{Introduction}

Unless the usefulness of tactile feedback in comparison with visual feedback still has to be fully explored $[2,3,4,9,10,13]$, there is some evidence for the integration of this form of communication. In [15] an attempt has been made to investigate the effectiveness of tactile feedback, using a computer mouse. The study shows that tactile feeback can provide an enhancement to visual stimuli for sighted individuals. For the visually impaired, the tactile feedback has the potential to become a partial substitute for visual stimuli.

Motivated by these results several universitary and non-universitary research institutions have worked on the development of tactile input devices mostly based on a computer mouse. All of the input devices described below combine tactile feedback with a pointing device. Some of them even provide force feedback. The tactile feedback of the multi-modal mouse [1] is provided by a needle which is pushed out of a hole in the mouse button directly under the user's finger. The tactile feedback of the multi-modal mouse is binary, i. e. either the needle stands out from the mouse button or it does not. This mouse has force feedback, too. However, it affects moving the whole mouse device, but not pressing the mouse button.

Another mouse input device with tactile feedback [6] has vibrating items on the left and right side of the mouse. Whether the left or the right or both items vibrate, depends on whether the mouse pointer is located to the left or right side of an object on the screen. The vibration frequency depends on the distance between mouse pointer and object. This mouse input device therefore simplifies positioning. As an improvement, the inventors tried to represent a part of the screen on the mouse button. For example the mouse button is a 8 by 3 matrix where every field represents one pixel of the screen. The user can thus feel a tiny part of the screen as a relief on the mouse button. Again, there is no force feedback intended to affect pressing the mouse button.

Hewlett Packard works on a computer system providing tactile and vibrational feedback on several kinds of input devices [7]. This computer system is basically based on stylus and touch screen as input devices although the same principle seems to make sense for mouse input devices, too. The feedback may be a continuous or pulsed vibration. The frequency and amplitude may be varying according to the 
selected position. The tactile feedback given when a user selects a soft function key may be different from that given when an icon is selected. For certain items, e.g. buttons, the feedback may be such that a physical click is both heard and felt by the user. Texture can only be felt if the user's finger or stylus is moving across the surface of the device. Therefore textural vibration is zero if the finger or stylus is stationary. The speed of moving the finger or the stylus is a parameter which is used to generate the feedback.

IBM works on a mouse ball-actuating device with force and tactile feedback [8]. The mouse utilizes a ball that rotates (rubs against) two shafts orthogonal to each other. In general these are only used for position sensing. For force feedback small electromagnets are attached to these $\mathrm{X}$ - and $\mathrm{Y}$-axes. A host computer will provide a signal to the mouse when force feedback is desired. The signal determines the amount of current to be applied to the magnets to determine the resistance against moving the mouse along each axis. At full force, the mouse cannot be moved and the shafts act as a brake. Smaller resistances create various "feels". Different forces on each shaft allow movement in a specific direction. A momentary increase in resistance may act as a tactile indication to a user that an event has occurred. Actively changing the force feedback can provide an apparent "groove", or path of least resistance, which may assist the impaired. Tactile feedback may be provided as a "clicker", which can be felt and heard by the user. The click can occur once when stepping over a grid or as a continuous buzz when entering an illegal zone. A complex clicker can be implemented to simulate rolling the mouse over surfaces of various roughness.

As an alternative approach the JoyString [14] is a "T"-form handle which is mounted on three wires on each end. The wires are used to provide force feedback. Sensors on the end of each wire measure with how much force and speed the user twists the handle. These data are transmitted to the computer. Thus it is possible to move and steer for example robots. Special devices on the end of each wire can make it more difficult or at least impossible to pull a specific wire. This force feedback can be used to stop the user's interaction in a certain direction, if, for example the robot bumps against the wall.

A special kind of the VPL DataGlove [5] is able to give tactile feedback to the user. Small piezoelectronic items beneath the fingertips of the gloves provide a tactile feelable tingling (20 to $40 \mathrm{~Hz}$ sinus). This simulates the touching of virtual objects. The intensity of this impression (according to the force the object is grabbed) can be varied by changing the frequency of the piezoelectronic items.

However all the devices mentioned so far are either too sophisticated and expensive for the common user or too limited in the feedback they can provide. The FeelMouse (see also [11], [12]) is the first device which provides tactile and force feedback with a reasonable tradeoff between cost and performance.

\section{A New Approach: The FeelMouse}

The FeelMouse is a mouse interaction device which gives the user tactile information and force feedback through changing the force which is necessary to push down the mouse button by a lifting magnet.

Usually users keep holding their fingers on the mouse button while interacting with the computer. Therefore, they feel the changing force as a kind of resistance which grows or falls. As every object may have a corresponding value of force, the 
resistance conveys the impression of touching objects of different softness. When the force is high enough, the finger is lifted up, when the force falls, the finger is lowered again. This gives users a three dimensional impression of the objects. They feel objects which jut out more or less from the virtual computer surface. The user therefore can recognize edges, borders, shapes or states of different objects.

By changing the resistance of the mouse button the force feedback is generated. This resistance is different for different objects or operations initiated by some screen elements. Some objects or operations may even be so "hard" that it takes much effort to push down the mouse button. This makes it possible to restrain users from selecting operations with protected objects or to prevent them from selecting operations, without any undo-possibilities and maybe terrible effects, if chosen in the wrong moment.

Depending on the application the required maximum force has to be high enough to generate an adequate number of graduations of force. The number of graduations has to be fixed, so that the difference in force can be clearly distinguished. It would seem useful if these graduations were logarithmic rather than linear. It would then be possible to recognize each of them more easily, as the next level of resistance is twice as strong as the previous (Fig. 1).

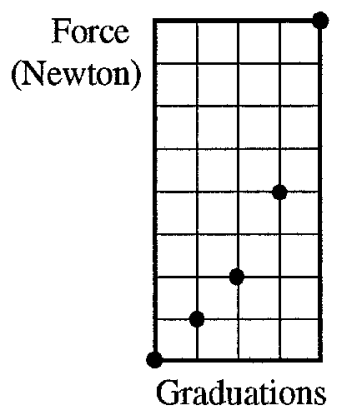

Fig. 1: Logarithmic graduations of force

In a usual mouse, the distance between the lowest (pressed down) and highest (not pressed down) position of the mouse button is very short. In the FeelMouse, there is a small lifting magnet attached beyond the mouse button. It is therefore possible to vary the distance as well as the resistance of the mouse button. On the one hand, the user should be able to feel the force long enough to recognize the changing graduation. On the other hand, the resistance perceived by the user should be as springy as possible. For both purposes it is important to keep the force upright over the total distance.

When the lifting magnet is activated, the magnet's anchor is pulled into the magnetic field. The force necessary to push the anchor out again will decrease the more the anchor is pushed out. In the above mentioned case a very strong magnet is necessary, as the force should not decrease beyond a certain resistance.

Another possibility is, to use a magnet with reverse behavior. This means, that the resistance remains constant or is even increasing, the more the anchor is pushed out of the magnetic field. This can be shown as magnetic lifting force curve as in fig. 2. 


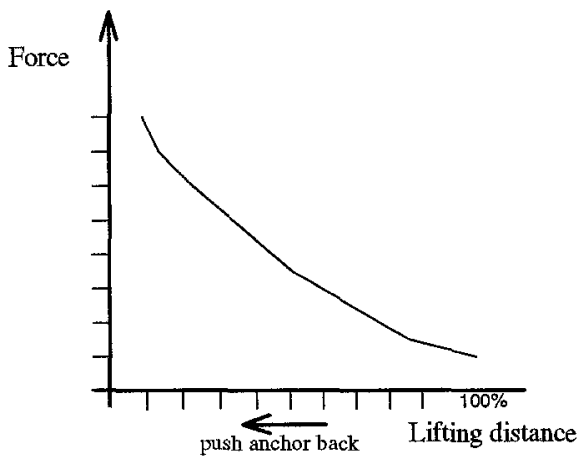

Fig. 2: Reverse magnetic lifting force curve

\section{Making GUIs Feelable}

How can the tactile feedback of the new device be explored to make the structure of a GUI feelable? The FeelMouse as a new device allows communication between man and machine in two directions at the same time. The information the computer gives to users is primarily tactile feedback based on input manipulations of the users. The users feel this feedback as a certain amount of force at the mouse button. As different objects or functions have their distinct amount of force, users can already recognize the object when they point at it. Besides, they can recognize whether a certain operation might be allowed for this object or not.

What distinguishes the FeelMouse from conventional mouse input devices on the application side is, that it generates a three dimensional image of the flat visual appearance of the GUT. Moreover it adds different tactile attributes like softness or roughness to the elements of the GUI. These are the basic thoughts when improving existing elements (i.e. objects and functions) of window management systems.

A very important objective in designing GUIs is to exclude as many potential sources of error as possible. In order not to tempt the user, only those functions should be offered which are valid for the very context. In menu boxes valid functions often are black, invalid ones are grayed out. This is an adequate visual distinction. But how can the visually impaired users be supported by this mechanism?

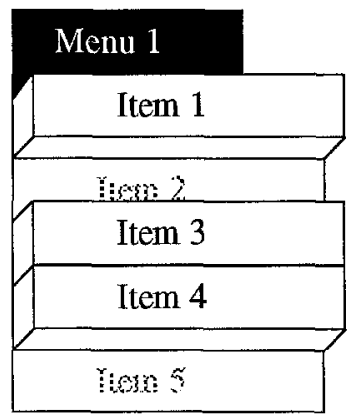

Fig. 3: Visual and tactile different menu items

Menu entries seem to be nothing else but buttons, combined in groups having similar functionality. An other way to distinguish valid from invalid functions is to add tactile attributes to these buttons. When a real button gets pressed, one has to use 
more or less effort to overcome the resistance of the button. By assigning differently high resistances to different states it is possible to recognize the state - valid or not by simply pointing at the button. Fig. 3 shows menu items, which can be distinguished visually (color) and tactily. The force is shown as differently high levels of 3-D representations of the button.

A valid menu item (button) can therefore be selected (pressed) with normal effort, i.e. the force necessary to press the FeelMouse-button is on the average level. Invalid items cannot be selected, because the resistance of the FeelMouse-button is very high. This means for the user that this function cannot be carried out in this particular context. The high resistance of the FeelMouse-button symbolizes a kind of lock to the user, although the button could be pressed using very high effort.

If the resistance depends on whether the function can be carried out or not, it is also possible to provide no resistance for invalid menu items instead of a very high one. This means, if the very function does not exist for a particular context, there cannot be a button for it.

As another example for the utilization of the special features of the FeelMouse in human-computer interaction a dialogue box, which is a combined interaction technique for selecting multiple elements of a selection set, can be applied. All input parameters for a certain function are combined into a clearly arranged box, where users can easily manipulate them for their purposes. After changing the parameters, the box must be closed explicitly. Therefore there are often two buttons with different effects. When selecting the button often called "O.K." or "Done" the dialogue box is closed and the function will be carried out with the selected parameter. The other button is mostly called "Cancel" and selecting it closes the box without carrying out the function.

A severe problem seems to be a selection of the "O.K" button by mistake. This happens, when users only have visual feedback. Expert users do interactions faster than windows are drawn on the screen or even automatically, without looking at the screen at all. That means that they possibly click buttons without reading their labels, just knowing where to click. Moments of thoughtlessness - also with non expert users - can therefore cause severe troubles, especially when the UNDO-function cannot be applied.

In order to focus one's attention to a potential error before it happens, it is necessary to make differently severe effects of buttons feelable. Users are able to feel the difference, for instance of the "O.K." and "Cancel" button, before they even select one of them. The force necessary to select the "O.K." button may then be much higher than the force for pressing the "Cancel" button. If users expect the normal resistance of the one, but in fact feel the high resistance of the other button, they will certainly think twice before carrying out the action.

An even more impressive form of reminding the user is to cause a striking tactile impression by rapidly changing the force of the resistance so that a vibration is generated. This is possibly the most impressive form of reminding the user of critical interactions. 


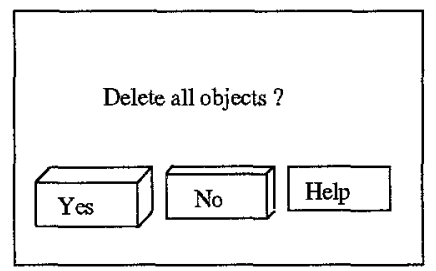

Fig. 4: Dialogue box with tactile buttons

Fig. 4. shows different buttons of a menu box. The more the three dimensional view of a button juts out from the box, the harder it is to press the FeelMouse button in order to select the appropriate function. Force feedback even makes it possible to exclude unauthorized users from selecting special functions. If the resistance of the FeelMouse button is maximum, it is equivalent to a lock, as described in fig. 4

A first approach to make graphic objects feelable is to transmit the visual represented three-dimensional appearance through tactile feedback to the user's haptic channel. Differently high elements produce differently high resistances of the FeelMouse button. This results in a realistic feelable impression of a tactile profile of the GUI. If one imagines the mouse pointer on the screen to be the virtual finger of the user, one may also think of a pixel of the screen as the mouse button. In this case it is possible for users to feel a single pixel and therefore to make very exact manipulations.

When users move the cursor across objects, they can feel their edges and surfaces. Fig. 5 shows lines on a surface. Along the inserted mouse move path, the user can feel the lines standing out from the surface like a relief (fig. 6a) or like being imprinted in stone (fig. 6b). In both cases it is very easy for the user to realize if the cursor points exactly to the object or not.

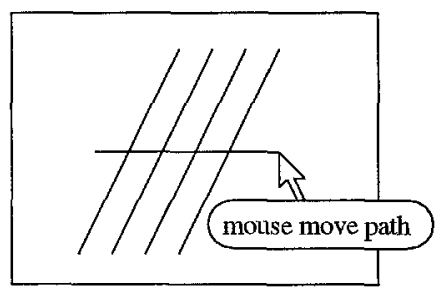

Fig. 5: Graphics example

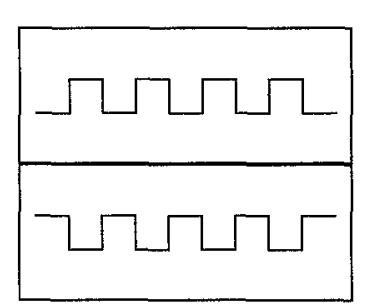

Fig. 6a,b: Tactile profiles of mouse move path

Fig. 7 shows a simple text editor with a GUI. It allows saving, quitting and inserting text at an arbitrary position by moving the cursor. How can the new interaction techniques provided by the FeelMouse be applied to make the functionality supplied by this editor feelable? 


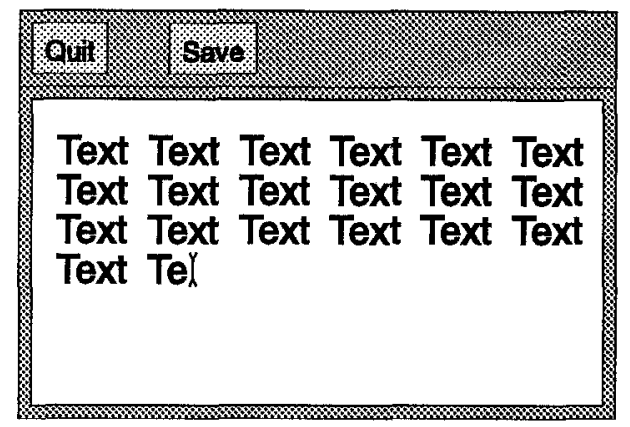

Fig. 7: Tactile user interface

"Quit" of course is a most critical function, which should not be selected accidentally, especially if the work has not been saved before. Therefore the feedback of the mouse consists of a vibrating button with a high amplitude. If there exists the danger of losing previous edits, the mouse button should also require very high force to be pressed. "Save" only is a useful action if there have been made changes to the text before. Therefore the function should be realized by the user by the high mouse button. If saving the edits makes sense there should be medium force required to press the button, if not, there should be no force resistance at all as nothing happens if a document with no changes is saved. To allow the visually impaired users to detect when they are moving the cursor into the area where text can be edited, the border of the text edit area must be feelable by a very high mouse button when crossing it. When the mouse is moved across a written word its button should vibrate with medium amplitude and low frequence. When reaching the end of the text and therefore clicking with the mouse button places the cursor correctly to append text, the mouse button should vibrate with high frequency to indicate the "hot spot". In this way a graphical interface could also be used by heavily visually impaired persons.

\section{Conclusions}

Visually impaired can be assisted in the use of computers in many different ways. One can enlarge certain areas of the screen depending on necessity. Research and development of voice interaction with the computer has come a long way. But research in the field of tactile input devices shows the importance of tactile cues for visually impaired persons. The FeelMouse has added to these support systems for handicapped computer users by providing tactile and force feedback. The FeelMouse makes it easier to orientate oneself on the screen by means of feeling. Computer users are now able to form a three dimensional image through the FeelMouse button on the objects on a two dimensional screen. Further research with the FeelMouse will include improved prototypes with a better magnet than used in the current prototype. User studies will clear up the still unsolved question of how many graduations of force a user can distinguish. It has to be taken into account that visually impaired persons probably have a better developed tactile sensitivity than non impaired users.

Despite of the many still unsolved questions experiences have shown that the FeelMouse is a valuable low cost input device for easier and more efficient interaction between man and machine. 


\section{References}

[1] Akamatsu M., Sato S.: The Multi-Modal Integrative Mouse - A Mouse With Tactile Display. ACM CHI'92 Poster and Short Talks, p. 60.

[2] Bach-y-rita, P., Tactile vision substitution: past and future, International Journal Neuroscience, 1983, Vol. 19, pp. 29-36.

[3] Collins, C. C., Madey, J. M. J., Tactile sensory replacement, Proceedings of the San Diego Biomedical Symposium, 1974, 13, pp. 15-26.

[4] Craig. J. C., Tactile pattern perception and its perturbations, Journal of Acoustical Society of America, 77 (1), January 1985, pp. 238-246.

[5] Foley J., van Dam A., Feiner S., Hughes J.: Computer Graphics, Principles and Practice. Addison Wesley, 1990, p. 355.

[6] Göbel M.: Eingabevorrichtung mit taktilem Feedback. Offenlegungschrift DE 4140780 A1, 1992, Deutsches Patentamt.

[7] Hewlett Packard Comp.: Input device with tactile feedback. WO-A192/00559, 1992. PCT.

[8] IBM: Mouse ball-actuating device with force and tactile feedback. IBM Disclosure Bulletin vol. 32, no. 9B, p. 234, New York, 1990.

[9] Jansson, G., Tactile guidance of movement, International Journal Neuroscience, 1983 , vol. 19, pp. 37-46.

[10] Kaczmarek, K., Bach-y-rita, P., Tompkins, W., Webster, J., A tactile visionsubstitution system for the blind: computer-controlled partial image sequencing, IEEE Transactions on Biomedical Engineering, vol. BME-32, No. 8, Aug. 1985, pp. 602-608.

[11] Penz, F., Kerstner, W. The feelmouse. In WWDU'92 Work With Display Units, Abstractbook from the Third International Conference on Work With Display Units, September 1-4, Berlin, 1992, p. P-22.

[12] Penz, F., Tscheligi, M. The FeelMouse: An Interaction Device with Force Feedback. In INTERCHI'93 Adjunct Proceedings: Bridges Between Worlds. ACM, 1993, pp. 121-122.

[13] Stark, L., Neurological control Systems: Studies in Bioengineering, 1968, pp. 297-337, 348-399

[14] Tello E.: Between Man and Machine. Byte Sept. 1988, p. 288.

[15] Terry J. A., Hsiao H.: Tactile Feedback In A Computer Mouse. Proc. of the 14th annual northeast bioengineering conference, 1988, Durham, NH, US, p. 146. 